\title{
Refined Verlinde formulas for Hilbert schemes of points and moduli spaces of sheaves on K3 surfaces
}

\author{
Lothar Göttsche
}

\begin{abstract}
We compute generating functions for elliptic genera with values in line bundles on Hilbert schemes of points on surfaces. As an application we also compute generating functions for elliptic genera with values in determinant line bundles on moduli spaces of sheaves on K3 surfaces.
\end{abstract}

Keywords. Hilbert schemes; moduli spaces of sheaves; elliptic genus; Verlinde formula

2020 Mathematics Subject Classification. 14C05; 14J60; 14J42; 58J26

[Français]

Formules de Verlinde raffinées pour les schémas de Hilbert de points et espaces de modules sur les surfaces $\mathrm{K} 3$

Résumé. Nous calculons les fonctions génératrices pour les genres elliptiques à valeur dans les fibrés en droites sur les schémas de Hilbert des points sur les surfaces. En guise d'application, nous calculons également les fonctions génératrices pour les genres elliptiques à valeurs dans les fibrés en droites sur les espaces de modules de faisceaux sur les surfaces K3.

Received by the Editors on March 13, 2019, and in final form on July 17, 2020.

Accepted on August 19, 2020.

Lothar Göttsche

International Centre for Theoretical Physics, Strada Costiera 11, 34100 Trieste, Italy

e-mail: gottsche@ictp.it

(C) by the author(s) 


\section{Contents}

1. Introduction. . . . . . . . . . . . . . . . . . . . . . . . . . . . . . 2

2. Background material . . . . . . . . . . . . . . . . . . . . . . . . . . . . 4

2.1. Hilbert schemes of points . . . . . . . . . . . . . . . . . . . . . . . . . . 4

2.2. Elliptic genus.. . . . . . . . . . . . . . . . . . . . . . . . . . . . . . . 5

2.3. Beauville-Bogomolov quadratic form . . . . . . . . . . . . . . . . . . . . . 5

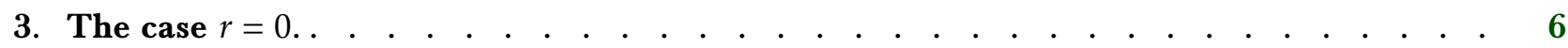

4. The case of Hilbert schemes of points on K3 surfaces

5. Moduli of sheaves on K3 surfaces . . . . . . . . . . . . . . . . . . . . . . . 10

References. . . . . . . . . . . . . . . . . . . . . . . . . . . 12

\section{Introduction}

The celebrated Verlinde formula (see [Ver88, NR93, BL94, Fal94]) is a formula for the generating function for dimensions of spaces of sections of line bundles on moduli spaces of vector bundles on algebraic curves.

Now let $S$ be a smooth projective surface over $\mathbb{C}$ and $S^{[n]}$ the Hilbert scheme of $n$ points on $S$. For every vector bundle $V$ on $S$ there is a corresponding tautological bundle $V^{[n]}$ of $\operatorname{rank} \operatorname{rk}\left(V^{[n]}\right)=n \operatorname{rk}(V)$, whose fibre over $Z \in S^{[n]}$ is $H^{0}\left(Z,\left.V\right|_{Z}\right)$. The map $V \mapsto V^{[n]}$ extends to a homomorphism from the Grothendieck group $K^{0}(S)$ of vector bundles on $S$ to $K^{0}\left(S^{[n]}\right)$. For $L \in S^{[n]} \operatorname{denote} \mu(L):=\operatorname{det}\left(\left(L-\mathcal{O}_{S}\right)^{[n]}\right)$ and $E:=\operatorname{det}\left(\mathcal{O}_{S}^{[n]}\right)$. Then it is well known that $\operatorname{Pic}\left(S^{[n]}\right)=\mu(\operatorname{Pic}(S)) \oplus \mathbb{Z E}$. The analogue of the Verlinde formula for Hilbert schemes of points is a formula for the generating function for holomorphic Euler characteristics $\chi\left(S^{[n]}, \mu(L) \otimes E^{r}\right)$. In [EGL01] such a formula was proven in the cases $r=-1,0,1$ or $K_{S}^{2}=K_{S} L=0$. On the other hand the celebrated Dijkgraaf-Moore-Verlinde-Verlinde formula [DMVV97], shown in [BL00, BL03, BL05], relates the generating function of the elliptic genera $\operatorname{Ell}\left(S^{[n]}\right)$ of Hilbert schemes of points to Siegel modular forms.

In this short note we interpolate between these two results, by proving a formula for $\operatorname{Ell}\left(S^{[n]}, \mu(L) \otimes E^{r}\right)$, the elliptic genus with values in the line bundle $\mu(L) \otimes E^{r}$. To state these results we introduce the following power series.

$$
\begin{aligned}
\phi_{-2,1}(q, y) & :=\left(y^{1 / 2}-y^{-1 / 2}\right)^{2} \prod_{n>0} \frac{\left(1-q^{n} y\right)^{2}\left(1-q^{n} / y\right)^{2}}{\left(1-q^{n}\right)^{4}}, \\
\wp(q, y) & :=\frac{1}{12}+\frac{1}{\left(y^{1 / 2}-y^{-1 / 2}\right)^{2}}+\sum_{n>0} \sum_{d \mid n} d\left(y^{d}-2+y^{-d}\right) q^{n}, \\
\phi_{0,1}(q, y) & :=12 \wp(x, y) \phi_{-2,1}(x, y), \\
h(q, y) & :=-\frac{1}{12}+\sum_{n>0} \sum_{d \mid n} \frac{n}{d}\left(y^{d}+y^{-d}\right) x^{n} .
\end{aligned}
$$

$\wp(q, y)$ is the Weierstrass $\wp$-function. It is standard that

$$
\operatorname{Ell}(S)=-(\wp+h) \phi_{-2,1} K_{S}^{2}+\phi_{0,1} \chi\left(\mathcal{O}_{S}\right) .
$$


We also introduce the following Borcherds type lifts. For $f:=\sum_{m, n} c_{m, n} q^{m} y^{n} \in \mathbb{Q}[[y, q]]$, we put

$$
\begin{aligned}
\mathbf{L}(f, p) & :=\prod_{l>0, m \geq 0, n \in \mathbb{Z}}\left(1-p^{l} q^{m} y^{n}\right)^{c_{l m, n},} \\
\mathbf{L}^{(a, b)}(f, p) & :=\prod_{l>0, m \geq 0, n \in \mathbb{Z}}\left(1-p^{l} q^{m} y^{n}\right)^{l^{a} n^{b} c_{l m, n}}, \quad a, b \in \mathbb{Z} .
\end{aligned}
$$

Then the DMVV formula says

$$
\sum_{n \geq 0} \operatorname{Ell}\left(S^{[n]}\right) p^{n}=\frac{1}{\mathbf{L}(\operatorname{Ell}(S), p)}=\frac{\mathbf{L}\left((\wp+h) \phi_{-2,1}, p\right)^{K_{S}^{2}}}{\mathbf{L}\left(\phi_{0,1}, p\right)^{\chi\left(\mathcal{O}_{S}\right)}} .
$$

The first theorem deals with the case $r=0$.

Theorem 1.1. Let $S$ be an algebraic surface, $L \in \operatorname{Pic}(S)$. Then

$$
\sum_{n \geq 0} \operatorname{Ell}\left(S^{[n]}, \mu(L)\right) p^{n}=\frac{\left(\mathbf{L}^{(2,0)}\left(-\phi_{-2,1}, p\right)\right)^{\frac{L^{2}}{2}}\left(\mathbf{L}^{(1,1)}\left(-\phi_{-2,1}, p\right)\right)^{\frac{L K_{S}}{2}}}{\mathbf{L}(\operatorname{Ell}(S), p)} .
$$

Specializing to $L=\mathcal{O}_{S}$, we recover the DMVV formula. Specializing to $q=0$ yields an infinite product formula for the $\chi_{y}$-genera with values in a $\mu(L)$, which in turn recovers for $L=\mathcal{O}_{S}$ the formula for the $\chi_{-y}$-genera of Hilbert schemes from [GS93]. We write

$$
\widetilde{\Delta}(p, y):=\prod_{n>0}\left(1-p^{n}\right)^{20}\left(1-p^{n} y\right)^{2}\left(1-p^{n} / y\right)^{2}, \quad \bar{\eta}(p)=\prod_{n>0}\left(1-p^{n}\right) .
$$

\section{Corollary 1.2 .}

$$
\sum_{n \geq 0} \bar{\chi}_{-y}\left(S^{[n]}, \mu(L)\right) p^{n}=\prod_{n>0}\left(\frac{\left(1-p^{n}\right)^{2}}{\left(1-p^{n} y\right)\left(1-\frac{p^{n}}{y}\right)}\right)^{\frac{n^{2}}{2} L^{2}} \prod_{n>0}\left(\frac{1-\frac{p^{n}}{y}}{1-p^{n} y}\right)^{\frac{n}{2} L K_{S}} \frac{\bar{\eta}(p)^{K_{S}^{2}}}{\widetilde{\Delta}(p, y)^{\frac{\chi\left(\mathcal{O}_{S}\right)}{2}}} .
$$

For general line bundles on Hilbert schemes of points we can partially determine the generating function.

Theorem 1.3. For every $r \in \mathbb{Z}$ there are universal power series $A_{r}, B_{r} \in \mathbb{Q}\left[y^{ \pm 1}\right][[z, q]]$ such that for every smooth projective surface $S$ and every $L \in \operatorname{Pic}(S)$ we have

$$
\sum_{n \geq 0} \operatorname{Ell}\left(S^{[n]}, \mu(L) \otimes E^{r}\right) p^{n}=\frac{\mathbf{L}^{(2,0)}\left(-\phi_{-2,1}, z\right)^{\frac{1}{2}\left(L^{2}+r^{2} \chi\left(\mathcal{O}_{S}\right)\right)} A_{r}^{L K_{S}} B_{r}^{K_{S}^{2}}}{\left(\mathbf{L}\left(2 \phi_{0,1}, z\right)\left(1-r^{2} z \frac{\partial}{\partial z} \log \mathbf{L}^{(2,0)}\left(\phi_{-2,1}, z\right)\right)\right)^{\frac{\chi\left(\mathcal{O}_{S}\right)}{2}}},
$$

with the change of variables $p=z \mathbf{L}^{(2,0)}\left(-\phi_{-2,1}, z\right)^{r^{2}}$.

Specialising to $q=0$ again yields a formula for the $\chi_{-y}$-genus with values in $\mu(L) \otimes E^{r}$.

Corollary 1.4. For every smooth projective surface $S$ and every $L \in \operatorname{Pic}(S)$ we have

$$
\begin{aligned}
\sum_{n \geq 0} \bar{\chi}_{-y}\left(S^{[n]}, \mu(L) \otimes E^{r}\right) p^{n} & =\prod_{n>0}\left(\frac{\left(1-z^{n}\right)^{2}}{\left(1-z^{n} y\right)\left(1-z^{n} / y\right)}\right)^{\frac{n^{2}}{2}\left(L^{2}+r^{2} \chi\left(\mathcal{O}_{S}\right)\right)} \\
& \times \frac{A_{r}(y, z, 0)^{L K_{S}} B_{r}(y, z, 0)^{K_{S}^{2}}}{\left(\widetilde{\Delta}(z, y)\left(1+r^{2} \sum_{n \geq 1}\left(y^{d}-2+y^{-d}\right) \frac{n^{3}}{d^{3}} z^{n}\right)\right)^{\frac{\chi\left(\mathcal{O}_{S}\right)}{2}}} .
\end{aligned}
$$

with the change of variables $p=z \prod_{n>0}\left(\frac{\left(1-z^{n}\right)^{2}}{\left(1-z^{n} y\right)\left(1-z^{n} / y\right)}\right)^{n^{2} r^{2}}$. 
Finally we show an analogue of Theorem 1.3 for moduli spaces of sheaves on K3 surfaces $S$. Fix $s \in \mathbb{Z}_{>0}$. Let $H$ be an ample line bundle on $S$ and $M_{S}^{H}\left(s, c_{1}, c_{2}\right)$ the moduli space of $H$-semistable sheaves on $S$ of rank $s$ with Chern classes $c_{1}, c_{2}$. We assume that $M_{S}^{H}\left(s, c_{1}, c_{2}\right)$ consists only of stable sheaves. For any choice of $s, c_{1}, c_{2}$, we denote

$$
\operatorname{vd}=\operatorname{vd}\left(s, c_{1}, c_{2}\right):=2 s c_{2}-(s-1) c_{1}^{2}-2\left(s^{2}-1\right) .
$$

Let $r \in \mathbb{Z}$. Let $L \in \operatorname{Pic}(S) \otimes \mathbb{Q}$ with $c_{1}(L)-\frac{r}{s} c_{1} \in H^{2}(S, \mathbb{Z})$. If $s$ divides $\left(c_{1}(L)-\frac{r}{s} c_{1}\right) c_{1}+r\left(\frac{c_{1}^{2}}{2}-c_{2}\right)$, we define a determinant line bundle $\mu(L) \otimes E^{r} \in \operatorname{Pic}\left(M_{S}^{H}\left(s, c_{1}, c_{2}\right)\right)$. This is the generalization of the line bundle with the same name on $S^{[n]}=M_{S}^{H}(1,0, n)$. We obtain the following result.

Theorem 1.5. Let $S$ be a $K 3$ surface, $s \in \mathbb{Z}_{>0}$. Under the assumptions above we have

$$
\operatorname{Ell}\left(M_{S}^{H}\left(s, c_{1}, c_{2}\right), \mu(L) \otimes E^{r}\right)=\operatorname{Coeff}_{p^{\mathrm{vd} / 2}}\left[\frac{\mathbf{L}^{(2,0)}\left(-\phi_{-2,1}, z\right)^{\frac{L^{2}}{2}+\frac{r^{2}}{s^{2}}}}{\mathbf{L}\left(2 \phi_{0,1}, z\right)\left(1-\frac{r^{2}}{s^{2}} z \frac{\partial}{\partial z} \log \mathbf{L}^{(2,0)}\left(\phi_{-2,1}, z\right)\right)}\right],
$$

with the change of variables $p=z \mathbf{L}^{(2,0)}\left(-\phi_{-2,1}, z\right)^{\frac{r^{2}}{s^{2}}}$, and

$$
\bar{\chi}_{-y}\left(M_{S}^{H}\left(s, c_{1}, n\right), \mu(L) \otimes E^{r}\right)=\operatorname{Coeff}_{p^{v d} / 2}\left[\frac{\prod_{n>0}\left(\frac{\left(1-z^{n}\right)^{2}}{\left(1-z^{n} y\right)\left(1-z^{n} / y\right)}\right)^{n^{2}\left(\frac{L^{2}}{2}+\frac{r^{2}}{s^{2}}\right)}}{\left(\widetilde{\Delta}(z, y)\left(1+\frac{r^{2}}{s^{2}} \sum_{n \geq 1} \sum_{d \mid n}\left(y^{d / 2}-y^{-d / 2}\right)^{2} \frac{n^{3}}{d^{3}} z^{n}\right)\right)}\right],
$$

with the change of variables $p=z \prod_{n>0}\left(\frac{\left(1-z^{n}\right)^{2}}{\left(1-z^{n} y\right)\left(1-z^{n} / y\right)}\right)^{\frac{n^{2} r^{2}}{s^{2}}}$.

In the special case of K3 surfaces Theorem 1.5 in particular confirms the conjectures of [GKW] about refinements of Verlinde formulas for moduli spaces of rank 2 sheaves on surfaces in the case of K3 surfaces. The specialization $L=\mathcal{O}_{X}$ reproduces in the case of K3 surfaces the formulas of [GK] on the elliptic genus of moduli spaces of sheaves on surfaces.

Acknowledgements. I thank Don Zagier for helping me with the proof of Lemma 4.3. This work grew out of collaboration with Martijn Kool. I thank him for many useful discussions.

\section{Background material}

\subsection{Hilbert schemes of points}

Let $S$ be a smooth projective surface. We denote $S^{[n]}$ the Hilbert scheme of $n$ points on $S$. It is a smooth projective variety of dimension $2 n$. Let $S^{(n)}$ be the $n$-th symmetric power of $S$. The Hilbert-Chow morphism $\pi: S^{[n]} \rightarrow S^{(n)}, Z \mapsto \operatorname{supp}(Z)$, sending a zero dimensional scheme to its support with multiplicities is a crepant resolution of $S^{(n)}$, i.e. it is birational and $\pi^{*} K_{S^{(n)}}=K_{S^{[n]}}$. Let $Z_{n}(S) \subset S \times S^{[n]}$ be the universal subscheme, with projections $p: Z_{n}(S) \rightarrow S^{[n]}, q: Z_{n}(S) \rightarrow S$. For a vector bundle $V$ of rank $r$ on $S$ the corresponding tautological vector bundle is $V^{[n]}:=p_{*} q^{*} V$, a vector bundle of rank $r n$ on $S^{[n]}$. This extends to a homomorphism ${ }^{[n]}: K^{0}(S) \rightarrow K^{0}\left(S^{[n]}\right)$ between the Grothendieck groups of vector bundles. We put $E:=\operatorname{det}\left(\mathcal{O}_{S}^{[n]}\right)$, and for a line bundle $L$ on $S$ we put $\mu(L):=\operatorname{det}\left(\left(L-\mathcal{O}_{S}\right)^{[n]}\right)$. Let $\eta: S^{n} \rightarrow S^{(n)}$ be the natural projection. Let $L_{n}:=\eta_{*}\left(\otimes_{i=1}^{n} p r_{i}^{*} L\right)^{\mathfrak{S}_{n}}$ be the $\mathfrak{S}_{n}$-equivariant pushforward, where $p r_{i}: S^{n} \rightarrow S$ is the $i$-th projection. Then it is well-known that $\mu(L)=\eta^{*}\left(L_{n}\right)$, and from the definitions it follows that $\operatorname{det}\left(V^{[n]}\right)=\mu(\operatorname{det}(V)) \otimes E^{\mathrm{rk}(V)}$. 


\subsection{Elliptic genus.}

For a compact complex manifold $M$, the $\chi_{-y}$-genus is

$$
\chi_{-y}(M):=\sum_{p}(-y)^{p} \chi\left(M, \Omega_{M}^{p}\right)
$$

Usually we consider the normalized version $\bar{\chi}_{-y}(M):=(-y)^{-\frac{\operatorname{dim}(M)}{2}} \chi_{-y}(M)$. For $V \in K^{0}(M)$ the $\chi_{-y}$-genus with values in $V$ is

$$
\bar{\chi}_{-y}(M, V):=y^{-\frac{\operatorname{dim}(M)}{2}} \sum_{p}(-y)^{p} \chi\left(M, \Omega_{M}^{p} \otimes V\right) .
$$

For a rank $r$ vector bundle $V$ on $M$ put

$$
\Lambda_{t} V:=\sum_{n=0}^{r}\left[\Lambda^{n} V\right] t^{n}, \quad \operatorname{Sym}_{t} V:=\sum_{n=0}^{\infty}\left[\operatorname{Sym}^{n} V\right] t^{n} .
$$

Write $y=e^{2 \pi i z}, q=e^{2 \pi i \tau}$. Then for $W \in K^{0}(M)$, the elliptic genus and the elliptic genus with values in $W$ are defined by

$$
\begin{aligned}
\operatorname{Ell}(M) & :=\operatorname{Ell}(M, z, \tau):=\bar{\chi}_{-y}\left(M, \mathcal{E}\left(T_{M}\right)\right), \\
\operatorname{Ell}(M, W) & :=\operatorname{Ell}(M, W, z, \tau):=\bar{\chi}_{-y}\left(M, \mathcal{E}\left(T_{M}\right) \otimes W\right),
\end{aligned}
$$

with

$$
\mathcal{E}(V):=\bigotimes_{n=1}^{\infty} \Lambda_{-y q^{n}} V^{\vee} \otimes \Lambda_{-y^{-1} q^{n}} V \otimes \operatorname{Sym}_{q^{n}}\left(V \oplus V^{\vee}\right)
$$

Let

$$
\theta(z, \tau):=-i q^{\frac{1}{8}}\left(y^{\frac{1}{2}}-y^{-\frac{1}{2}}\right) \prod_{n>0}\left(1-q^{n}\right)\left(1-q^{n} y\right)\left(1-q^{n} / y\right)
$$

be the classical Jacobi theta function, where we write $y=e^{2 \pi i z}, q=e^{2 \pi i \tau}$. Let $c(M)=\prod_{j=1}^{n}\left(1+x_{j}\right)$ be a formal splitting of the total Chern class of M. Putting

$$
\operatorname{ELL}(M):=\operatorname{ELL}(M, z, \tau)=\prod_{j} x_{j} \frac{\theta\left(\frac{x_{j}}{2 \pi i}-z, \tau\right)}{\theta\left(\frac{x_{j}}{2 \pi i}, \tau\right)} \in H^{*}(X, \mathbb{Q})\left[y^{ \pm \frac{1}{2}}\right][[q]],
$$

it follows from the definitions and the Riemann-Roch theorem that

$$
\operatorname{Ell}(M)=\int_{M} \operatorname{ELL}(M), \quad \operatorname{Ell}(M, W)=\int_{M} \operatorname{ELL}(M) \operatorname{ch}(W) .
$$

\subsection{Beauville-Bogomolov quadratic form}

Let $X$ be a compact holomorphic symplectic manifold of dimension $\operatorname{dim}(X)=2 \mathrm{~m}$. We briefly review some properties of the Beauville-Bogomolov quadratic form $q_{X}: H^{2}(X) \rightarrow \mathbb{Q}$ on $X$ from [Huy99, Sections 1.9-1.11]. Note that the odd Chern classes of $X$ vanish.

Theorem 2.1. For any $\beta \in H^{4 k}(X, \mathbb{Q})$ in the sub-algebra generated by the Chern classes of $X$, there is a constant $c(\beta) \in \mathbb{Q}$, such that for all $\alpha \in H^{2}(X, \mathbb{Q})$

$$
\int_{X} \beta \alpha^{\operatorname{dim}(X)-2 k}=c(\beta) q_{X}(\alpha)^{m-k}
$$

The quantity $c(\beta)$ is invariant under deformation of $X$. 
Corollary 2.2. There exists a polynomial $h_{X}=h_{X}(z)$ with coefficients in $\mathbb{Q}\left[y^{ \pm 1}\right][[q]]$ such that

$$
\int_{X} \operatorname{ELL}(X) \exp (\alpha)=h_{X}\left(q_{X}(\alpha)\right), \quad \text { for all } \alpha \in H^{2}(X, \mathbb{Z})
$$

The polynomial $h$ is invariant under deformation of $X$.

\section{The case $r=0$.}

In this section we will prove Theorem 1.1. We start by reviewing some of the ideas and definitions of [BL05]. For a Kawamata $\log$-terminal pair $(Z, D)$ of a projective variety and a divisor $D$ in $Z$ with an action of a finite group $G$, Borisov and Libgober define in [BL05, Definition 3.6] the orbifold elliptic class $\mathrm{Ell}_{\mathrm{orb}}(Z, D, G)=\mathrm{Ell}_{\mathrm{orb}}(Z, D, G, z, \tau)$. In fact they first define it in [BL05, Definition 3.2] in case $X$ is nonsingular and the pair $(X, E)$ is also $G$-normal (see [BL05, Definition 3.1] for the definitions) by an explicit formula. In the general case, they define in [BL05, Definition 3.6] $\operatorname{lll}_{\text {orb }}(Z, D, G):=\rho_{*} \operatorname{Ell}_{\text {orb }}(X, E, G)$ for $\rho:(X, E) \rightarrow(Z, D)$ a $G$-normal equivariant resolution. We will write $\operatorname{Ell}_{\text {orb }}(Z, G), \operatorname{Ell}_{\text {orb }}(Z, D), \operatorname{Ell}_{\text {orb }}(Z)$ in case $D=0$ and/or $G$ is the trivial group.

If $X$ is a nonsingular projective variety, with an action of a finite group their formula specializes to

$$
\operatorname{Ell}_{\mathrm{orb}}(X, G):=\frac{1}{|G|} \sum_{g h=h g} \sum_{Z}[Z]\left(\prod_{\lambda(g)=\lambda(h)=0} \prod_{j} x_{\lambda, j}\right) \prod_{\lambda, j} \frac{\theta\left(\frac{x_{\lambda, j}}{2 \pi i}+\lambda(g)-\tau \lambda(h)-z\right)}{\theta\left(\frac{x_{\lambda, j}}{2 \pi i}+\lambda(g)-\tau \lambda(h)\right)} e^{2 \pi i \lambda(h) z} .
$$

Here $Z$ runs over the irreducible components of the common fixpoint set of $g$ and $h$, and $[Z]$ is the class of $Z$ in the Chow group of $X$. The restriction of $T_{X}$ to $Z$ splits into linearized bundles according to the $[0,1)$-valued characters $\lambda$ of $\langle g, h\rangle$. We denote by $x_{\lambda, j}$ the elements of a formal splitting of the total Chern class of the bundle with character $\lambda$. If $G$ acts effectively on $(Z, D)$ and $(Z / G, D / G)$ is the quotient pair in the sense of [BL05, Definition 2.7], they show in [BL05, Theorem. 5.3] that $\psi_{*} \operatorname{Ell}_{\text {orb }}(Z, D)=\operatorname{Ell}_{\text {orb }}(Z / G, D / G)$ for the quotient morphism $\psi: Z \rightarrow Z / G$. This is in particular true for the pairs $(Z, 0),(Z / G, 0)$, if $Z$ is nonsingular and $G$ is acting freely in codimension 1 .

Now consider the action of $\mathfrak{S}_{n}$ on $S^{n}$, and recall the quotient morphism $\eta: S^{n} \rightarrow S^{(n)}$ and the Hilbert Chow morphism $\pi: S^{[n]} \rightarrow S^{(n)}$. As $S^{[n]}$ is nonsingular we have $\operatorname{ELL}\left(S^{[n]}\right)=\operatorname{Ell}_{\text {orb }}\left(S^{[n]}\right)$. As $\pi$ is a crepant resolution, [BL05, Theorem 3.5] implies that $\pi_{*} \operatorname{ELL}\left(S^{[n]}\right)=\operatorname{Ell}_{\text {orb }}\left(S^{(n)}\right)$. Thus we find by the above

$$
\pi_{*} \operatorname{ELL}\left(S^{[n]}\right)=\operatorname{Ell}_{\text {orb }}\left(S^{(n)}\right)=\eta_{*} \operatorname{Ell}_{\text {orb }}\left(S^{n}, \mathfrak{S}_{n}\right)
$$

Now let $L$ be a line bundle on $S$, then we have

$$
\begin{aligned}
\operatorname{Ell}\left(S^{[n]}, \mu(L)\right) & =\int_{S^{[n]}} \operatorname{ELL}\left(S^{[n]}\right) \operatorname{ch}(\mu(L)) \\
& =\int_{S^{(n)}} \pi_{*}\left(\operatorname{ELL}\left(S^{[n]}\right)\right) \operatorname{ch}\left(L_{n}\right) \\
& =\int_{S^{n}} \operatorname{Ell}_{\text {orb }}\left(S^{n}, \mathfrak{S}_{n}\right) \operatorname{ch}\left(\sum_{i=1}^{n} p r_{i}^{*} L\right) .
\end{aligned}
$$

In the second line we have used $\pi^{*}\left(L_{n}\right)=\mu(L)$ and the projection formula, and in the third line we have used (3.2), $\eta^{*}\left(L_{n}\right)=\otimes_{i=1}^{n} p r_{i}^{*} L$ and again the projection formula. 
Let $x_{1}, x_{2}$ be the Chern roots of $T_{S}$. Then

$$
\begin{aligned}
& \operatorname{Ell}(S, L)=\int_{S}\left(1+L+\frac{L^{2}}{2}\right) \prod_{j=1}^{2} x_{i} \frac{\theta\left(\frac{x_{j}}{2 \pi i}-z, \tau\right)}{\theta\left(\frac{x_{j}}{2 \pi i}, \tau\right)} \\
& =\operatorname{Ell}(S)-\frac{K_{S} L}{2} \operatorname{Coeff}_{x^{1}}\left(x \frac{\theta\left(\frac{x}{2 \pi i}-z, \tau\right)}{\theta\left(\frac{x}{2 \pi i}, \tau\right)}\right)^{2}+\frac{L^{2}}{2} \operatorname{Coeff}_{x^{2}}\left(x \frac{\theta\left(\frac{x}{2 \pi i}-z, \tau\right)}{\theta\left(\frac{x}{2 \pi i}, \tau\right)}\right)^{2} \\
& =\operatorname{Ell}(S)+\frac{K_{S} L}{2} y \frac{\partial}{\partial y} \phi_{-2,1}+\frac{L^{2}}{2} \phi_{-2,1} .
\end{aligned}
$$

Now we prove Theorem 1.1 by adapting the proof of [BL05, Theorem 6.1]. Note that in the notations of [BL05] we have $D=0$, which leads to many simplifications.

Let $(g, h)$ be a commuting pair in $\mathfrak{S}_{n}$. We sum up the description of the action of $g, h$ and their fixpoint sets in the proof of [BL05, Theorem 6.1]. We have a decomposition $\{1, \ldots, n\}=J_{1} \cup \ldots \cup J_{m}$ into the orbits of the subgroup generated by $g, h$. Thus the action of $(g, h)$ on $S^{n}$ restricts to an action on each of the corresponding products $S^{J_{l}}$. Furthermore we can write $\left|J_{l}\right|=a_{l} b_{l}$ for positive integers $a_{l}, b_{l}$, and up to reordering of the elements of $J_{l}$ the action of $(h, g)$ on $S^{J_{l}}$ can be described as follows. Write

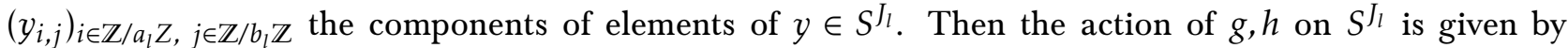
$h\left(y_{i, j}\right)=y_{i, j+1}, g\left(y_{i, j}\right)=y_{i+1, j}$ for $0 \leq i<a_{l}-1, g\left(y_{a_{l}-1, j}\right)=y_{0, j+s}$, for some $s \in\left\{0, \ldots, b_{l}-1\right\}$, and $s$ determines the action of $(g, h)$ on $J_{l}$ uniquely. The fixpoint set $\left(S^{J_{l}}\right)^{g, h}$ is $S$ embedded via the diagonal map $j_{l}: S \rightarrow S^{J_{l}}$.

Changing their notation slightly, we denote for $(a, b, s):=\left(a_{l}, b_{l}, s_{l}\right)$ by

$$
F_{a, b, s}:=j_{l}^{*}\left(\left(\prod_{\lambda(g)=\lambda(h)=0} \prod_{j} x_{\lambda, j}\right) \prod_{\lambda, j} \frac{\theta\left(\frac{x_{\lambda, j}}{2 \pi i}+\lambda(g)-\tau \lambda(h)-z\right)}{\theta\left(\frac{x_{\lambda, j}}{2 \pi i}+\lambda(g)-\tau \lambda(h)\right)} e^{2 \pi i \lambda(h) z}\right)
$$

the pullback of the contribution of the restriction of the pair $(f, g)$ to $\operatorname{Ell}_{\text {orb }}\left(S^{J_{l}}, \mathfrak{S}_{J_{l}}\right)$ in (3.1) multiplied by $a ! b !$. Then it is shown in [BL05, Lemma 6.4] that

$$
F_{a, b, s}=\prod_{j=1,2} \frac{x_{j} \theta\left(\frac{a x_{j}}{2 \pi i}-a z, \frac{a \tau-s}{b}\right)}{\theta\left(\frac{a x_{j}}{2 \pi i}, \frac{a \tau-s}{b}\right)} .
$$

Note that the left hand side is

$$
\frac{1}{a^{2}} \prod_{j=1,2} \frac{a x_{j} \theta\left(\frac{a x_{j}}{2 \pi i}-a z, \frac{a \tau-s}{b}\right)}{\theta\left(\frac{a x_{j}}{2 \pi i}, \frac{a \tau-s}{b}\right)}=\sum_{k=0}^{2} a^{k-2} \operatorname{ELL}\left(S, a z, \frac{a \tau-s}{b}\right)_{k},
$$

where ()$_{k}$ denotes the part in degree $k$. As $j_{l}^{*} \eta^{*}\left(L_{n}\right)=L^{a b}$, we obtain

$$
\begin{aligned}
\int_{S} F_{a, b, s} \operatorname{ch}\left(j_{l}^{*} \eta^{*}\left(L_{n}\right)\right) & =\int_{S}\left(\sum_{k=0}^{2} a^{k-2} \operatorname{ELL}\left(S, a z, \frac{a \tau-s}{b}\right)_{k}(a b)^{2-k} \operatorname{ch}_{2-k}(L)\right) \\
& =\operatorname{Ell}\left(S, L^{b}, a z, \frac{a \tau-s}{b}\right) .
\end{aligned}
$$

By definition it is clear that the contribution of $(g, h)$ to $\int_{S^{n}} \operatorname{Ell}_{\mathrm{orb}}\left(S^{n}, \mathfrak{S}_{n}\right) \eta^{*} L_{n}$ is

$$
\frac{1}{n !} \prod_{l=1}^{m} \int_{S} F_{a_{l}, b_{l}, S_{l}} j_{l}^{*} \eta^{*}\left(L_{n}\right) \text {. }
$$

Thus arguing as after [BL05, Lemma 6.6], writing

$$
\operatorname{Ell}\left(S^{[n]}\right)=\sum_{l, n} c_{l, n} y^{l} q^{n}, \quad \phi_{-2,1}=\sum_{l, n} d_{l, n} y^{l} q^{n}
$$


and using (3.3) in the third line, we obtain

$$
\begin{aligned}
\sum_{n \geq 0} \operatorname{Ell}\left(S^{[n]}, \mu(L)\right) p^{n}=\sum_{n \geq 0} p^{n}\left(\int_{S^{n}} \operatorname{Ell}_{\operatorname{orb}}\left(S^{n}, \mathfrak{S}_{n}\right) \eta^{*} L_{n}\right) \\
\quad=\exp \left(\sum_{a, b>0} \sum_{s=0}^{b-1} \frac{p^{a b}}{a b} \operatorname{Ell}\left(X, L^{b}, a z, \frac{a \tau-s}{b}\right)\right) \\
=\exp \left(\sum_{a, b>0} \sum_{m, l} \sum_{s=0}^{b-1}\left(c_{m, l}+\left(b l \frac{L K_{S}}{2}+b^{2} \frac{L^{2}}{2}\right) d_{m, l}\right) \frac{p^{a b}}{a b} y^{a l} q^{\frac{a m}{b}} e^{2 \pi i \frac{m s}{b}}\right) \\
=\exp \left(\sum_{a, b>0} \sum_{m, l}\left(c_{m b, l}+\left(b l \frac{L K_{S}}{2}+b^{2} \frac{L^{2}}{2}\right) d_{m b, l}\right) \frac{p^{a b}}{a} y^{a l} q^{a m}\right) \\
=\prod_{b=1}^{\infty} \prod_{m, l}\left(1-p^{b} y^{l} q^{m}\right)^{-c_{m b, l}-l b d_{m b, l} \frac{K_{S} L}{2}-b^{2} d_{m b, l} \frac{L^{2}}{2}} .
\end{aligned}
$$

This proves Theorem 1.1.

To deduce Corollary 1.2 from Theorem 1.1 , we note that $\bar{\chi}_{-y}\left(S^{[n]}, \mu(L)\right)=\left.\operatorname{Ell}\left(S^{[n]}, \mu(L)\right)\right|_{q=0}$, and by definition $\left.\mathbf{L}(f, p)\right|_{q=0}=\mathbf{L}\left(\left.f\right|_{q=0}, p\right)$. Thus we have

$$
\begin{aligned}
\left.\mathbf{L}\left(-\phi_{-2,1}, p\right)\right|_{q=0} & =\mathbf{L}\left(-y+2-y^{-1}, p\right)=\prod_{n>0} \frac{\left(1-p^{n}\right)^{2}}{\left(1-y p^{n}\right)\left(1-y^{-1} p^{n}\right)}, \\
\left.\mathbf{L}^{(2,0)}\left(-\phi_{-2,1}, p\right)\right|_{q=0} & =\prod_{n>0}\left(\frac{\left(1-p^{n}\right)^{2}}{\left(1-y p^{n}\right)\left(1-y^{-1} p^{n}\right)}\right)^{n^{2}}, \\
\left.\mathbf{L}^{(1,1)}\left(-\phi_{-2,1}, p\right)\right|_{q=0} & =\mathbf{L}^{(1,0)}\left(y^{-1}-y, p\right)=\prod_{n>0}\left(\frac{1-y^{-1} p^{n}}{1-y p^{n}}\right)^{n}, \\
\left.\mathbf{L}\left((\wp+h) \phi_{-2,1}, p\right)\right|_{q=0} & =\mathbf{L}(1, p)=\bar{\eta}(p) \text { and }\left.\mathbf{L}\left(\phi_{0,1}, p\right)\right|_{q=0}=\mathbf{L}\left(y+10+y^{-1}\right)=\Delta(y, p)^{1 / 2} .
\end{aligned}
$$

\section{The case of Hilbert schemes of points on K3 surfaces}

Now we want to consider the case of Hilbert schemes of points on K3 surfaces. We obtain a formula for all $r$.

Proposition 4.1. Let $S$ be a K3 surface and $L \in \operatorname{Pic}(S)$. Then

$$
\operatorname{Ell}\left(S^{[n]}, \mu(L) \otimes E^{r}\right)=\operatorname{Coeff}_{p^{n}}\left[\left(\mathbf{L}^{(2,0)}\left(-\phi_{-2,1}, p\right)\right)^{\frac{L^{2}}{2}-r^{2}(n-1)} \mathbf{L}\left(-2 \phi_{0,1}, p\right)\right] .
$$

Proof. The Hilbert scheme $S^{[n]}$ is a holomorphic symplectic manifold; let $q_{S^{[n]}}$ be its Beauville-Bogolomov quadratic form. By Corollary 2.2 there exists a polynomial $h_{S^{[n]}}(z)$ with coefficients in $\mathbb{Q}\left[y^{ \pm 1}\right][[q]]$ such that

$$
\operatorname{Ell}\left(S^{[n]}, M\right)=h_{S^{[n]}}\left(q_{S^{[n]}}\left(c_{1}(M)\right)\right), \quad \text { for all } M \in \operatorname{Pic}\left(S^{[n]}\right) .
$$

It is shown in [Bea83, lem. 9.1] that for $L \in \operatorname{Pic}(S)$ we have

$$
q_{S^{[n]}}(L+r E)=L^{2}-2 r^{2}(n-1) .
$$

Therefore Proposition 4.1 follows from Theorem 1.1. 
Now we want to deduce Theorem 1.3 from Proposition 4.1. We know

$$
\operatorname{Ell}\left(S^{[n]}, \mu(L) \otimes E^{r}\right)=\int_{S^{[n]}} \operatorname{ELL}\left(S^{[n]}\right) \operatorname{ch}\left(\mu(L) \otimes E^{r}\right),
$$

where ELL is the genus associated to a power series, and $\mu(L) \otimes E^{r}=\operatorname{det}\left(\left(L+(r-1) \mathcal{O}_{S}\right)\right)^{[n]}$. Therefore [EGL01, Theorem 4.2] applies and gives the following.

Corollary 4.2. For every $r \in \mathbb{Z}$, there are universal power series $F_{r, 1}, F_{r, 2}, F_{r, 3}, F_{r, 4} \in \mathbb{Q}\left[y^{ \pm 1}\right][[q, p]]$ such that for every smooth projective surface $S$ and every $L \in \operatorname{Pic}(S)$ we have

$$
\sum_{n \geq 0} \operatorname{Ell}\left(S^{[n]}, \mu(L) \otimes E^{r}\right) p^{n}=F_{r, 1}^{L^{2} / 2} F_{r, 2}^{L K_{S} / 2} F_{r, 3}^{K_{S}^{2}} F_{r, 4}^{\chi\left(\mathcal{O}_{S}\right)}
$$

Using Corollary 4.2, in order to prove Theorem 1.3, we only need show the formulas for $F_{r, 1}$ and $F_{r, 4}$, which are determined by their values for $S$ a K3 surface. So let again $S$ be a K3 surface, and $L \in \operatorname{Pic}(S)$. Then by Proposition 4.1 we get

$$
\sum_{n \geq 0} \operatorname{Ell}\left(S^{[n]}, \mu(L) \otimes E^{r}\right) p^{n}=\sum_{n \geq 0} p^{n} \operatorname{Coeff}_{p^{n}}\left[\left(\mathbf{L}^{(2,0)}\left(-\phi_{-2,1}\right)(p)^{\frac{L^{2}}{2}-(n-1) r^{2}} \mathbf{L}\left(-2 \phi_{0,1}\right)(p)\right)\right] .
$$

Thus Theorem 1.3 follows from the following lemma.

Lemma 4.3. Let $g(p)=1+\sum_{i \geq 1} a_{i} p^{i}$ be a power series starting with 1 , let $f(p)$ be a power series. Fix $w, k \in \mathbb{Q}$. Put

$$
h(p):=\sum_{n \geq 0} p^{n} \operatorname{Coeff}_{p^{n}}\left[g(p)^{w-k n} f(p)\right]
$$

Then

$$
h(p)=\frac{g(z)^{w} f(z)}{1+k z \frac{d}{d z} \log (g(z))} \quad \text { where } p=z g(z)^{k} .
$$

Proof. Without loss of generality we can assume that $w=0$ (otherwise replace $f(p)$ by $g(p)^{w} f(p)$ ), and $k=1$ (otherwise replace $g(p)$ by $g(p)^{k}$ and note that $z \frac{d}{d z} \log \left(g(z)^{k}\right)=k z \frac{d}{d z} \log (g(p))$ ). We can describe $h(p)$ as follows: for a variable $u$ write $g(p)^{-u} f(p)=\sum_{n \geq 0} h_{n}(u) p^{n}$, with $h_{n}(u)$ a polynomial in $u$, then $h(p)=\sum_{n \geq 0} h_{n}\left(p \frac{d}{d p}\right) p^{n}$, i.e. move all factors of $u$ to the left and then replace $u$ by $p \frac{d}{d p}$. We make the variable transformation $p=e^{x}$, so that $p \frac{p}{d p}=\frac{d}{d x}$, and we write $g(p)=e^{a(x)}, f(p)=\phi(x)$. Then we obtain

$$
\begin{aligned}
h(p) & =\sum_{n \geq 0} \frac{(-1)^{n}}{n !} \frac{d^{n}}{d x^{n}}\left(a(x)^{n} \phi(x)\right) \\
& =\left.\frac{\phi(\eta)}{1+a^{\prime}(\eta)}\right|_{\eta=x-a(\eta)} \\
& =\left.\frac{f(z)}{1+z \frac{d}{d z} \log g(z)}\right|_{p=z g(z)} .
\end{aligned}
$$

In the second line we have used the Lagrange inversion formula

$$
\sum_{n=0}^{\infty} \frac{1}{n !} \frac{d^{n}}{d x^{n}}\left(a(x)^{n} f(x)\right)=\left.\frac{f(z)}{1-a^{\prime}(z)}\right|_{z=x+a(z)} .
$$

In the third line we put $z:=e^{\eta}$, thus $p=e^{x}=e^{\eta+a(\eta)}=z g(z)$. 


\section{Moduli of sheaves on K3 surfaces}

In this section we extend our results to moduli spaces of sheaves on K3 surfaces. First we briefly recall determinant line bundles on moduli spaces of sheaves, for details see e.g. [GNY09, Section 1.1], [HL10, Chapter 8].

For a Noetherian scheme $Y$ denote by $K(Y)$ and $K^{0}(Y)$ the Grothendieck groups of coherent sheaves and locally free sheaves. If $Y$ is nonsingular and projective, then $K(Y)=K^{0}(Y)$. We denote by $[\mathcal{F}]$ the class of a sheaf $\mathcal{F}$ in $K(Y)$. For a proper morphism $f: Z \rightarrow Y$ the pushforward $f_{!}: K(Z) \rightarrow K(Y)$ is defined by $f_{!}([\mathcal{F}]):=\sum_{i}(-1)^{i}\left[R^{i} f_{*} \mathcal{F}\right]$. For any morphism $f: Z \rightarrow Y$ the pullback $f^{*}: K^{0}(Y) \rightarrow K^{0}(Z)$ is defined by $f^{*}[\mathcal{F}]=\left[f^{*} \mathcal{F}\right]$ for $\mathcal{F}$ a locally free sheaf on $Y$.

Now let $S$ be a smooth projective surface. On $K(S)$ there is a quadratic form $(u, v) \mapsto \chi(S, u \otimes v)$ to be denoted by $\chi(u \otimes v)$. Classes $u, v \in K(S)$ are called numerically equivalent if $u-v$ is in the radical of this quadratic form. Let $K(S)_{\text {num }}$ be the set of numerical equivalence classes. Let $c \in K(S)_{\text {num }}$. For a flat family $\mathcal{E}$ of coherent sheaves on $S$ of class $c \in K(S)_{\text {num }}$ parametrized by a scheme $T$, let $q: S \times T \rightarrow S$, $p: S \times T \rightarrow T$ be the projections and define $\lambda_{\mathcal{E}}: K(S) \rightarrow \operatorname{Pic}(S)$ by the composition

$$
K(S) \stackrel{q^{*}}{\rightarrow} K^{0}(S \times T) \stackrel{\otimes[\mathcal{E}]}{\longrightarrow} K^{0}(S \times T) \stackrel{p_{1}}{\longrightarrow} K^{0}(T) \stackrel{\operatorname{det}^{-1}}{\longrightarrow} \operatorname{Pic}(T) .
$$

For $c \in K_{\text {num }}(S)$ let $K_{c}:=\{v \in K(S) \mid \chi(v \otimes c)=0\}$. Let $H \in \operatorname{Pic}(S)$ be ample, denote $M_{S}^{H}(c)$ the moduli space of $H$-semistable sheaves of class $c$. Assume that $H$ is general, i.e. if $\mathcal{F} \in M_{S}^{H}(c)$ is strictly $H$-semistable, then it is strictly semistable for all $H^{\prime}$ in a neighbourhood of $H$. Then there exists a homomorphism $\lambda: K_{c} \rightarrow \operatorname{Pic}\left(M_{S}^{H}(c)\right)$, such that if $\mathcal{E}$ is a flat family of coherent sheaves on $S$ of class $c$ parametrized by $T$, then $\phi_{\mathcal{E}}^{*}(\lambda(v))=\lambda_{\mathcal{E}}(v)$, where $\phi_{\mathcal{E}}: T \rightarrow M_{S}^{H}(c)$ is the classifying morphim associated to $\mathcal{E}$. For a class $v \in K(S)$ denote

$$
\operatorname{vd}(v):=2 \operatorname{rk}(v) c_{2}(v)-(\operatorname{rk}(v)-1) c_{1}(v)^{2}-\left(\operatorname{rk}(v)^{2}-1\right)
$$

the expected dimension of $M_{S}^{H}(v)$. We obtain the following result.

Proposition 5.1. Let $S$ be a K3 surface and $c \in K_{\text {num }}(S)$, with $r(c)>0$ or with $r(c)=0$ and $c_{1}(c)$ nef and big. Let $H$ ample on $S$ such that $M_{S}^{H}(c)$ only consists of stable sheaves. Assume furthermore $\operatorname{vd}(c)>1$. Then

$$
\begin{aligned}
\operatorname{Ell}\left(M_{S}^{H}(c), \lambda(v)\right) & =\mathrm{Coeff}_{p^{\frac{\mathrm{vd}(c)}{2}}}\left[\left(\mathbf{L}^{(2,0)}\left(-\phi_{-2,1}, p\right)\right)^{\frac{\mathrm{vd}(v)}{2}-1} \mathbf{L}\left(-2 \phi_{0,1}, p\right)\right], \\
\bar{\chi}_{-y}\left(M_{S}^{H}(c), \lambda(v)\right) & =\mathrm{Coeff}_{p^{\frac{\mathrm{v}(c)}{2}}}\left[\frac{1}{\widetilde{\Delta}(p, y)} \prod_{n>0}\left(\frac{\left(1-p^{n}\right)^{2}}{\left(1-p^{n} y\right)\left(1-p^{n} / y\right)}\right)^{n^{2}\left(\frac{\mathrm{vd}(v)}{2}-1\right)}\right] .
\end{aligned}
$$

Proof. We adapt the arguments in [GNY09, Section 1.5]. The Mukai lattice of $S$ is $H^{*}(S, \mathbb{Z})$ with the symmetric bilinear form

$$
\left\langle w, w^{\prime}\right\rangle=\int_{S}\left(c_{1} c_{1}^{\prime}-r a^{\prime}-r^{\prime} a\right), \quad w=\left(r, c_{1}, a\right), w^{\prime}=\left(r^{\prime}, c_{1}^{\prime}, a^{\prime}\right) .
$$

Let $\phi: K(S) \rightarrow H^{*}(S, \mathbb{Z})$ be the homomorphism defined by $\phi(E)=\operatorname{ch}(E) \sqrt{\operatorname{td}(S)}$. Let $w:=\phi(c)$, then $\phi$ induces a injective homomorphism $\phi: K_{c} \rightarrow w^{\perp}$. There is a homomorphism $\theta_{w}: w^{\perp} \rightarrow H^{2}\left(M_{S}^{H}(c), \mathbb{Z}\right)$, such that $\theta_{w}(\phi(v))=c_{1}(\lambda(v))$ for all $v \in K_{c}$. We have assumed that $\operatorname{rk}(c)>0$ or that $\operatorname{rk}(c)=0$ and $c_{1}(c)$ is nef and big, furthermore $\operatorname{vd}(c)>1$, and $M_{S}^{H}(c)$ consists only of stable sheaves. Under these assumptions we know ([GNY09, Theorem 1.14], [Yos01a], [Yos01b]), that $M_{S}^{H}(c)$ is an irreducible symplectic manifold which is deformation equivalent to $S^{[\operatorname{vd}(c) / 2]}, \theta_{w}: w^{\perp} \rightarrow H^{2}\left(M_{S}^{H}(c), \mathbb{Z}\right)$ is surjective, and for $x$ in $w^{\perp}$ we have $\langle x, x\rangle=q_{M_{S}^{H}(c)}\left(\theta_{w}(x)\right)$. 
Let $v \in K_{c}$. By Corollary 2.2 we have

$$
\operatorname{Ell}\left(M_{S}^{H}(c), \lambda(v)\right)=\int_{M_{S}^{H}(c)} \operatorname{ELL}\left(M_{S}^{H}(c)\right) \exp \left(c_{1}(\lambda(v))\right)=h_{M_{S}^{H}(c)}\left(q_{M_{S}^{H}(c)}\left(\theta_{w}\left(c_{1}(\lambda(v))\right)\right)\right) .
$$

As $M_{S}^{H}(c)$ is deformation equivalent to $S^{[\mathrm{vd}(c) / 2]}$, we have $h_{M_{S}^{H}(c)}=h_{S[\mathrm{vd}(c) / 2]}$. We compute

$$
q_{M_{S}^{H}(c)}\left(\theta_{w}\left(c_{1}(\lambda(v))\right)\right)=\langle\phi(v), \phi(v)\rangle=\operatorname{vd}(v)-2 .
$$

Thus we get by Proposition 4.1 and (4.1),(4.2) that

$$
\operatorname{Ell}\left(M_{S}^{H}(c), \lambda(v)\right)=h_{S[\mathrm{vd}(c) / 2]}(\operatorname{vd}(v)-2)=\operatorname{Coeff}_{p^{\frac{\mathrm{vd}(c)}{2}}}\left[\mathbf{L}^{(2)}\left(-\phi_{-2,1}, p\right)^{\frac{\mathrm{vd}(v)}{2}-1} \mathbf{L}\left(-2 \phi_{0,1}, p\right)\right] .
$$

In order to express this formula in terms of generating functions we denote the line bundles $\lambda(v)$ on different moduli spaces $M_{S}^{H}(c)$ in a unified way, generalizing our notation on Hilbert schemes of points.

Notation 5.2. For fixed $c \in K(S)_{\text {num }}$ we write $s=\operatorname{rk}(c), c_{1}=c_{1}(c), c_{2}=c_{2}(c)$. Let us denote by $\operatorname{det}(c)$ and $\operatorname{det}(v) \in \operatorname{Pic}(S)$ the determinant line bundles. We assume that $s>0$, and denote $\operatorname{vd}:=\operatorname{vd}(c)$. Let $r \in \mathbb{Z}$. Let $L \in \operatorname{Pic}(S) \otimes \operatorname{det}(c)^{r / s}$, and denote $M:=L \otimes \operatorname{det}(c)^{-r / s} \in \operatorname{Pic}(S)$. If $s \operatorname{divides} M c_{1}+r\left(\frac{c_{1}^{2}}{2}-c_{2}\right)$, we define $\mu(L) \otimes E^{r} \in \operatorname{Pic}\left(M_{S}^{H}(c)\right) \in \operatorname{Pic}\left(M_{S}^{H}(c)\right)$ by $\mu(L) \otimes E^{r}:=\lambda(v)$ for $v \in K(S)$ with

$$
\operatorname{rk}(v)=r, \quad c_{1}(v)=M, \quad c_{2}(v)=\frac{M^{2}}{2}+2 r+\frac{M c_{1}}{s}+\frac{r}{s}\left(\frac{c_{1}^{2}}{2}-c_{2}\right) .
$$

Note that the condition on $c_{2}(v)$ is equivalent to $\chi(S, c \otimes v)=0$ i.e. to $v \in K_{c}$, so that $\mu(L) \otimes E^{r}$ is well-defined.

\section{Remark 5.3.}

(1) When $r=0$ this definition coincides with the definition of the Donaldson line bundle $\mu(L)$ (see e.g. [GNY09], [GKW]).

(2) When $s=1$ the definition specializes to that of $\mu(L) \otimes E^{r}$ on $S^{[n]}$ under the identification $S^{[n]}=$ $M_{S}^{H}\left(1, c_{1}, n\right)$, for any first Chern class $c_{1}$.

(3) If all sheaves in $M_{S}^{H}(c)$ are slope stable, then twisting by a line bundle $A$ gives an isomorphism $\phi_{A}: M_{S}^{H}(c) \rightarrow M_{S}^{H}(c \otimes A)$, and it is easy to see that $\phi_{A}^{*}\left(\mu(L) \otimes E^{r}\right)=\mu(L) \otimes E^{r}$.

Now we prove Theorem 1.5.

Proof of Theorem 1.5. Fix $c$ with $\operatorname{rk}(c)=s>0, c_{1}(c)=c_{1}, c_{2}(c)=c_{2}$, fix $r \in \mathbb{Z}$, let $L \in \operatorname{Pic}(S) \otimes \operatorname{det}(c)^{r / s}$, denote by $M:=L \otimes \operatorname{det}(c)^{-r / s}$ the corresponding element in $\operatorname{Pic}(S)$, and assume that $s$ divides $M c_{1}+r\left(\frac{c_{1}^{2}}{2}-c_{2}\right)$. Let $v \in K_{c}$ fullfill (5.1), so that $\mu(L) \otimes E^{r}=\lambda(v)$. Plugging the relations

$c_{1}(v)=c_{1}(L)-\frac{r}{s} c_{1}, \quad c_{2}(v)=\frac{c_{1}(v)^{2}}{2}+2 r+\frac{c_{1}(v) c_{1}}{s}+\frac{r}{s}\left(\frac{c_{1}^{2}}{2}-c_{2}\right) \quad$ and $\quad c_{2}=\frac{1}{2 s}\left(\operatorname{vd}(c)+(s-1) c_{1}^{2}+2\left(s^{2}-1\right)\right)$ into the formula

$$
\operatorname{vd}(v)=2 r c_{2}(v)-(r-1) c_{1}(v)^{2}-2\left(r^{2}-1\right)
$$

gives by direct computation

$$
\operatorname{vd}(v)=L^{2}+2-\frac{r^{2}}{s^{2}}(\operatorname{vd}(c)-2)
$$

Thus Proposition 5.1 gives

$$
\operatorname{Ell}\left(M_{S}^{H}(c), \mu(L) \otimes E^{r}\right)=\operatorname{Coeff}_{p^{v d}(c) / 2}\left[\left(\mathbf{L}^{(2,0)}\left(-\phi_{-2,1}, p\right)\right)^{\frac{L^{2}}{2}-\frac{r^{2}}{s^{2}}(\operatorname{vd}(c) / 2-1)} \mathbf{L}\left(-2 \phi_{0,1}, p\right)\right]
$$

Applying Lemma 4.3 gives Theorem 1.5. 


\section{References}

[Bea83] A. Beauville, Variétés kähleriennes dont la premiere classe de Chern est nulle, J. Diff.Geom. 18 (1983), 755-782.

[BL94] A. Beauville and Y. Laszlo, Conformal blocks and generalized theta functions, Comm. Math. Phys. 164 (1994), 385-419.

[BL00] L. A. Borisov and A. Libgober, Elliptic genera of toric varieties and applications to mirror symmetry, Invent. Math. 140 (2000), 453-485.

[BL03] _ Elliptic genera of singular varieties, Duke Math. Jour. 116 (2003), 319-351.

[BL05] _ _ McKay correspondence for elliptic genera, Annals of Math. 161 (2005), 1521-1569.

[Bor95] R. E. Borcherds, Automorphic forms on $O_{s+2,2}(\mathbb{R})$ and infinite products, Invent. Math. 120 (1995), 161-213.

[DMVV97] R. Dijkgraaf, G. Moore, E. Verlinde and H. Verlinde, Elliptic genera of symmetric products and second quantized strings, Commun. Math. Phys. 185 (1997), 197-209.

[EGL01] G. Ellingsrud, L. Göttsche, and M. Lehn, On the cobordism class of the Hilbert scheme of a surface, Jour. Alg. Geom. 10 (2001), 81-100.

[Fal94] G. Faltings, A proof of the Verlinde formula, J. Alg. Geom. 3 (1994), 347-374.

[GK] L. Göttsche and M. Kool, A rank 2 Dijkgraaf-Moore-Verlinde-Verlinde formula, Commun. Number Theory Phys. 13 (2019), No. 1, 165-201.

[GKW] L. Göttsche, M. Kool and Runako Williams, Verlinde formulas on complex surfaces I: K-theoretic invariants, preprint arXiv:1903.03869 (2019).

[GNY09] L. Göttsche, H. Nakajima, and K. Yoshioka, K-theoretic Donaldson invariants via instanton counting, Pure Appl. Math. Q. 5 (2009), 1029-1111.

[GS93] L. Göttsche, W. Soergel, Perverse sheaves and the cohomology of Hilbert schemes of smooth algebraic surfaces, Math. Ann. 296 (1993), 235-245.

[Huy99] D. Huybrechts, Compact hyper-Kähler manifolds: basic results, Invent. Math. 135 (1999), 63-113.

[HL10] D. Huybrechts, M. Lehn, The geometry of moduli spaces of sheaves, Cambridge University Press (2010).

[NR93] M. S. Narasimhan and T. R. Ramadas, Factorisation of generalised theta functions. I, Invent. Math. 114 (1993), 565-623.

[Ver88] E. Verlinde, Fusion rules and modular transformations in $2 d$ conformal field theory, Nucl. Phys., B 300 (1988), 360-376.

[Wit88] E. Witten, The index of the Dirac operator in loop space, in: Elliptic curves and modular forms in algebraic geometry, P. Landweber, ed., Springer-Verlag (1988), 161-181.

[Yos01a] K. Yoshioka, Moduli spaces of stable sheaves on abelian surfaces, Math. Ann. 321 (2001), 817-884.

[Yos01b] K. Yoshioka, A note on Fourier-Mukai transform, preprint arXiv:mathAG/0112267 (2001). 